\title{
Philosophiques
}

\section{Dominique Folscheid, La philosophie allemande de Kant à Heidegger, P.U.F., coll. " Premier Cycle ", Paris, 1993, 436 p.}

\section{Luc Langlois}

Volume 22, numéro 2, automne 1995

URI : https://id.erudit.org/iderudit/027357ar

DOI : https://doi.org/10.7202/027357ar

Aller au sommaire du numéro

Éditeur(s)

Société de philosophie du Québec

ISSN

0316-2923 (imprimé)

1492-1391 (numérique)

Découvrir la revue

Citer ce compte rendu

Langlois, L. (1995). Compte rendu de [Dominique Folscheid, La philosophie allemande de Kant à Heidegger, P.U.F., coll. « Premier Cycle », Paris, 1993, 436 p.] Philosophiques, 22(2), 545-547. https://doi.org/10.7202/027357ar d'utilisation que vous pouvez consulter en ligne.

https://apropos.erudit.org/fr/usagers/politique-dutilisation/ 
Dominique Folscheid, La philosophie allemande de Kant à Heidegger, P.U.F., coll. "Premier Cycle », Paris, 1993, 436 p.

\section{par Luc Langlois}

Le titre même de l'ouvrage trahit déjà toute la mesure de son ambition. Présenter en quatre cents pages à peine un continent aussi dense de l'histoire de la philosophie, dont les contours demandent d'ailleurs à être préalablement définis, relève en réalité de l'exploit et exige quelque audace. Dans l'ensemble, c'est-à-dire malgré les inégalités qu'entraîne inévitablement un tel travail d'équipe, on peut dire que l'entreprise fut fort honorablement menée et que cet outil saura rendre de précieux services à l'étudiant, pour qui il a été principalement conçu. L'immensité du projet requérait tout de même quelques précisions qui nous seront fournies par l'éditeur dans son texte de présentation. Ainsi le vocable "philosophie allemande ", qui ne veut se rabattre ni sur une catégorie d'ordre culturel ou géograp̧hique, ni sur le seul critère linguistique, sera entendu dans un sens assez large, qui permettra par exemple d'inclure Kierkegaard, influencé par l'Idéalisme de Hegel bien qu'il ait écrit en danois, tout en excluant par ailleurs Leibniz, qui appartient plutôt à la philosophie classique. Bref on aura cherché à cerner un certain " esprit " de la philosophie allemande (p. 4), ce qui ne va pas sans peine compte tenu de la disparité inouie des auteurs retenus.

À tout seigneur tout honneur, c'est à Kant qu'il reviendra d'inaugurer ce parcours historique. Si le texte de D. Folscheid est soucieux d'embrasser l'ensemble de la démarche kantienne telle que développée dans les trois Critiques, l'interprétation qui nous en est donnée laisse parfois songeur. Il est par exemple étonnant de lire que l'auteur des Fondements de la métaphysique des moeurs et de la Métaphysique des moeurs ait voulu disqualifier la métaphysique en morale (p. 29), là où précisément Kant croyait voir l'horizon de son redéploiement et de son relancement légitime, sur les décombres de la vieille métaphysique dogmatique. Toute l'équivoque vient peut-être de ce que l'A. ne souligne pas suffisamment la polysémie du terme "métaphysique " chez Kant, qu'il paraît réserver au rationalisme pré-critique alors qu'il englobe tout aussi bien la $C R P$ (que Kant aimait appeler une a métaphysique de la métaphysique », en tant que savoir a priori de l'a priori) que la métaphysique de la liberté qui prend sa source dans la raison pratique et connaît son épanouissement dans la Doctrine du Souverain Bien. Il sera tout de même fait mention à la p. 37 du réinvestissement kantien des thèmes séculaires de la métaphysique par le biais de la morale.

M. Vetö nous propose quant à lui une lecture de Fichte très stimulante, bien qu'elle n'avoue peut-être pas assez clairement sa filiation avec l'interprétation récente de $\mathrm{R}$. Lauth et A. Philonenko, intéressante mais qui est 
encore loin d'entraîner l'adhésion majoritaire. Quelques exemples. Les trois principes de la Doctrine de la science (p. 51) seront conçus comme des illusions transcendantales à portée simplement régulatrice, et perdront par conséquent tout caractère constitutif (pace Hegel!). La philosophie morale de Fichte fera fond sur une doctrine de l' « intersubjectivite » (p. 59), thème qui est pourtant d'apparition tardive et dont il n'est pas sûr qu'il ait beaucoup préoccupé les philosophes de l'Idéalisme allemand. Enfin, la médiation juridique sera considérée comme le seul et unique point de jonction entre raison théorique et raison pratique (p. 66) puisqu'en elle se traduit dans la nature un ordre de liberté en devenir et en progrès, d'où la place centrale des Fondements du droit naturel, appelés à soutenir tout l'édifice du système fichtéen (thèse déjà défendue par A. Renaut).

La philosophie de Schelling sera entre bonnes mains puisque JeanFrançois Marquet Liberté et existence. Étude sur la formation de la philosophie de Schelling en assurera la présentation, dans un texte d'autant plus remarquable qu'il réussit à vaincre la difficulté d'une pensée qui a la réputation d'avoir résisté à bien des tentatives de systématisation. Un choix éditorial bien avisé, parce que largement appuyé par l'historiographie, fera de Hegel le point d'aboutissement de l'Idéalisme allemand, malgré certaines relectures récentes qui voudraient réserver cet honneur à Schelling qui a survécu à son ancien condisciple (M. Frank).

Faisons un bond dans le temps pour signaler les deux fortes contributions de Françoise Dastur, l'une consacrée à la phénoménologie de Husserl, l'autre à l'ontologie fondamentale de Heidegger. La pensée de Husserl (qui a beaucoup écrit mais assez peu publié, comme on sait) a toujours eu ceci de déroutant qu'elle n'est jamais parvenue à trouver sa forme d'expression définitive et canonique, et a emprunté des directions qui ne paraissent pas toujours convergentes. Aussi l'utilité du travail de l'A. consistera entre autres à établir une périodisation qui permette d'apprécier l'évolution de cette auvre, découpée ici en trois séquences. La première va de la Philosophie de l'arithmétique de 1891 aux Recherches logiques de 1900-1901 qui marque l'abandon progressif du psychologisme caractérisant les premiers écrits jusqu'à la formulation initiale du programme de la phénoménologie. La seconde, qui débute en 1905 avec la « découverte " de la réduction phénoménologique et s'achève avec les Méditations cartésiennes de 1929 correspond à la période de constitution de la phénoménologie transcendantale et à l'orientation des recherches de Husserl dans le sens d'une science de l'égologie pure. La dernière philosophie, préoccupée par la crise des sciences et par l'élaboration du thème de la Lebenswelt, trouvera son aboutissement dans la Krisis de 1935-1936.

L'A. n'oubliera évidemment pas de relever les origines phénoménologiques de Heidegger, mais n'hésitera pas non plus à signaler le virage herméneutique que ce dernier imprimera à la phénoménologie dans Être et temps, ce que ne souligne pas toujours avec assez de vigueur la littérature secondaire francophone, relativement insensible à ce thème de l'herméneutique qui n'a 
pas marqué sa propre tradition autant qu'en Allemagne. La science sans présupposé prenant appui sur la conscience pure et impartiale que visait Husserl, fera ainsi place à la facticité d'un Dasein qui n'est jamais en relation d'extériorité par rapport aux phénomènes qui font encontre dans l'horizon temporel de son existence et dont l'appréhension est irrémédiablement livrée au cercle herméneutique. D'où la signification éminemment pratique accordée par Heidegger à l'ontologie, mais aussi à la phénoménologie qui constitue le moyen de son renouvellement et qui perdra les inflexions plus théorétiques que lui avait données Husserl.

Il aurait peut-être fallu continuer sur cette lancée, et accorder un meilleur traitement à l'herméneutique philosophique de Gadamer qui n'aura droit qu'à une page et demie, même si son empreinte sur la philosophie allemande d'après-guerre est profonde. $\grave{A}$ ce compte on aurait souhaité que Habermas, l'autre grande figure de cette philosophie allemande des dernières décennies, héritât d'un peu plus que des deux pages et demie qui lui sont consacrées. Ces distorsions dans la réception française des courants de pensée aujourd'hui dominants outre-Rhin sont certes regrettables, mais n'enlèvent rien à la bonne qualité d'ensemble de cet ouvrage, dont certaines des monographies constituent une source utile d'information.

Faculté de philosophie

Université Laval 